\title{
Anderson localization of classical waves in weakly scattering one-dimensional Levy lattices
}

\author{
Ara A. Asatryan and Alexander Novikov \\ School of Mathematical and Physical Sciences, University of Technology Sydney, Sydney, New South Wales 2007, Australia
}

(Received 1 September 2018; revised manuscript received 3 November 2018; published 21 December 2018)

\begin{abstract}
Anderson localization of classical waves in weakly scattering one-dimensional Levy lattices is studied analytically and numerically. The disordered medium is composed of layers with alternating refractive indices and with thickness disorder distributed according to the Pareto distribution $\sim 1 / x^{(\alpha+1)}$. In Levy lattices the variance (or both variance and mean) of a random parameter does not exist, which leads to a different functional form for the localization length. In this study an equation for the localization length is obtained, and it is found to be in excellent agreement with the numerical calculations throughout the spectrum. The explicit asymptotic equations for the localization lengths for both short and long wavelengths have been deduced. It is shown that the localization length tends to a constant at short wavelengths and it is determined by the layer interface Fresnel coefficient. At the long wavelengths the localization length is proportional to the power of the wavelength $\ell \sim \lambda^{\alpha}$ for $1<\alpha<2$, and it has a transcendental behavior $\ell \sim \lambda^{2} / \ln \lambda$ for $\alpha=2$. For $\alpha>2$, where the variance of the random distribution exists, the localization length attains its classical long-wavelength asymptotic form $\ell \sim \lambda^{2}$.
\end{abstract}

DOI: 10.1103/PhysRevB.98.235144

\section{INTRODUCTION}

The subject of wave propagation in disordered media continues to attract substantial interest. This is not surprising given its considerable importance for applications. This interest has been further spurred by the discovery of Anderson localization, which was established first for electronic waves [1] and later extended to waves of a general nature [2-7].

Anderson localization is a fascinating phenomenon in which the interference of multiply scattered waves in disordered media can lead to the localization of all states, which prohibits the wave's propagation and leads to the inhibition of wave transport. In such situations the amplitude of the incident wave decays exponentially. The scale on which this decay takes place is called the localization length $\ell$, which also describes the spatial spread of the modes of the system. It has been established for a while that in one-dimensional systems $[3,5,8,9]$ under quite general conditions all states become localized in the limit of infinite media [8], while the problem of the Anderson localization for higher-dimensional systems $d>1$ still remains unresolved [10].

Despite the number of rigorous results obtained for onedimensional systems $[8,9]$, these systems still continue to attract researchers, partly because of a desire to better understand different aspects of localization and partly to consider the effects on localization of different properties of random media. For example, the effects of absorption on Anderson localization have been considered in $[11,12]$, while the effects of gain have been considered in $[13,14]$. The properties of Anderson localization have been considered in different media as well, such as disordered periodic media $[15,16]$, metamaterials [17,18], graphene [19], and gyrotropic media [20]. It has been established that metamaterials can suppress Anderson localization. In this case, in contrast to the conventional asymptotic behavior of the localization length at long wavelengths [21] $l \sim \lambda^{2}$, it was shown that the localization length is proportional to $l \sim \lambda^{8}$ in [22-24].
The effects of the type of random distribution on Anderson localization have been considered also. An interesting case here is to consider the disorder with heavy-tail distributions. Such distributions may not have a second or even a first moment. Note that for one-dimensional systems the localization length at long wavelengths is inversely proportional to the variance of disorder, and it is clear that the functional form of the localization length $\ell$ will be different for Levy distributions where the variance does not exist. Note that here we will loosely refer to "Levy distribution" as any distribution with heavy tails which does not have a variance or mean. A particular example of this class of distributions is the Levy distribution [25].

The effects of Cauchy distribution on Anderson localization were studied in [26,27], while the conductance properties through quantum wires were considered in $[28,29]$. The effects of the long-range correlated potential on Anderson localization were considered in [30-34], while the effects of the Brewster anomaly were investigated numerically in [35].

A closely related question of photon propagation in Levy glasses was considered in [36]. Levy glasses are artificial materials in which scattering centers are Levy distributed. These materials have very interesting light propagation properties, where the transport can be superdiffusive. This can substantially influence the properties of Anderson localization in such materials. The question of Anderson localization for one-dimensional chains with heavy-tail disorder distribution is considered in [37].

In these studies only the general functional form of the localization length at long wavelengths was deduced for the long-wavelength limit or the localization length calculated numerically using Monte Carlo averaging.

The main aim of this paper is to obtain an equation for the localization length which is applicable for all wavelengths for a Levy-type disorder distribution and to present explicit asymptotic expressions for the localization length for both short and long wavelengths. 
Here, we study the properties of the photon localization in one-dimensional Levy disordered lattices. We assume that the Fresnel reflection coefficient $\rho_{j}$ between the adjacent layers is small $\left|\rho_{j}\right| \ll 1$ and derive a general equation for the localization length. All theoretical results are confirmed by Monte Carlo numerical simulations and are shown to be in excellent agreement. The short- and long-wavelength asymptotic equations for the localization length have been deduced.

In what follows, Sec. II presents the description of the model and its analytical solution. The derivations of the shortand long-wavelength asymptotic expressions for the localization length are presented in Sec. II C. The results of numerical calculations and the verification of the obtained analytical equations are presented in Sec. III.

\section{THEORETICAL CONSIDERATION}

\section{A. Description of the model}

One of the key characteristics of Anderson localization is the localization length $\ell$, which for one-dimensional problems can be calculated using the relation

$$
\ell=-\lim _{N \rightarrow \infty} \frac{2 L}{\ln \left|T_{N}\right|^{2}},
$$

where $T_{N}$ is the transmission coefficient of a stack of $N$ disordered layers and $L$ is the length of the overall stack. This quantity characterizes the "depth" of the propagation in which the wave's amplitude is exponentially reduced due to disorder. The localization length also characterizes the spatial spread of the modes of the corresponding random system. It has been proven that under quite general conditions the localization length is a self-averaging quantity, and as the stack length $L \rightarrow \infty$, the localization length is not random.

The self-averaging property of the localization length allows its numerical calculation using the transmission length defined by the relation

$$
l_{N}=-\frac{2 L}{\left\langle\ln \left|T_{N}\right|^{2}\right\rangle},
$$

where $L$ is the length of the finite stack and $T_{N}$ is its transmission coefficient and the brackets $\langle\cdots\rangle$ denote the averaging over the disorder. In the limit as the stack length $N \rightarrow \infty$, the transmission length coincides with the localization length,

$$
\lim _{N \rightarrow \infty} l_{N}=l \text {. }
$$

The stack length should be sufficiently long that the transmission length $l_{N}<L$ and it is independent of $L$.

In what follows, we consider weakly scattering stacks in which the Fresnel reflection coefficient at each interface is small, $\left|\rho_{j}\right| \ll 1$. This translates to a requirement that the refractive index contrast between the layers is small.

As a model of a disordered medium, we consider a stack composed of $N$ alternating nonmagnetic layers of two types with the refractive index $v_{A}$ and the refractive index $v_{B}$ (see Fig. 1). We will assume that $N$ is an even number, although this is not necessary. The layer thicknesses $d_{A}$ and $d_{B}$ and their refractive indices $v_{A}$ and $v_{B}$ can be random. However, we will consider here only the layer thickness disorder, and we

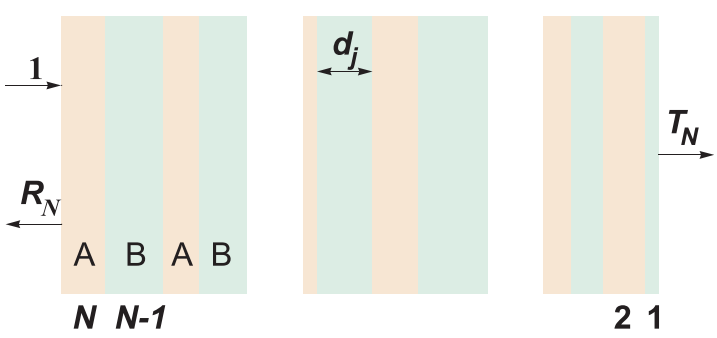

FIG. 1. The geometry of the problem.

will assume that the probability density distribution of layer thickness $d_{j}=x$ is given by a Pareto distribution

$$
f(x)=\frac{\alpha x_{m}^{\alpha}}{x^{\alpha+1}},
$$

where $x \geqslant x_{m}$ and $f(x)=0$ for $x<x_{m}$ and the parameter $\alpha>0$. This distribution belongs to the class of heavy-tail distributions. Furthermore, for the values $0<\alpha \leqslant 2$ the variance of the distribution is infinite, and for the values $0<\alpha \leqslant 1$ the mean of the distribution is also infinite. Following Ref. [37], we will restrict our consideration to values $1<\alpha \leqslant 2$, in which there exists the mean of the thickness distribution (4) given by

$$
\langle d\rangle=\frac{\alpha x_{m}}{\alpha-1} .
$$

We will also consider values for $\alpha>2$, where the variance of the distribution (4) exists and it is given by

$$
\operatorname{Var}(d)=\frac{\alpha x_{m}^{2}}{(\alpha-1)^{2}(\alpha-2)} .
$$

For the set of values $0<\alpha \leqslant 1$, where the mean of a layer thickness is infinite, the law of large numbers does not hold, and this requires a separate consideration.

For values $1<\alpha \leqslant 2$ this disordered structure represents a one-dimensional Levy glass [37] where there is an interesting interplay between the Anderson localization and an analog of Levy flights in one dimension. This inevitably leads to a different functional law of the localization length as a function of the wavelength. Indeed, at long wavelengths in the classical case the localization length is inversely proportional to the variance of disorder $\ell \sim \operatorname{Var}^{-1}(d)$, which is no longer valid for $1<\alpha \leqslant 2$.

To characterize the localization properties in such disordered lattices we first consider the numerical calculation of the transmission using a recursive procedure [22]. We calculate the total amplitude transmission and reflection coefficients of the stack in terms of the recurrence relations

$$
\begin{gathered}
T_{n}=\frac{T_{n-1} t_{n}}{1-R_{n-1} r_{n}}, \\
R_{n}=r_{n}+\frac{R_{n-1} t_{n}^{2}}{1-R_{n-1} r_{n}}
\end{gathered}
$$

for $n=2, \ldots, N$, in which both the input and output media are uniform media with the refractive index $v_{b}$ and with layers that are enumerated from $n=1$ at the rear of the stack through $n=N$ at the front. The initial values for the reflection and the transmission coefficients are $R_{0}=0$ and $T_{0}=1$. 
The single-layer amplitude transmission $t_{j}$ and reflection $r_{j}$ coefficients are given by

$$
\begin{aligned}
r_{j} & =\frac{\rho_{j}\left(1-e^{2 i \beta_{j}}\right)}{1-\rho_{j}^{2} e^{2 i \beta_{j}}}, \\
t_{j} & =\frac{\left(1-\rho_{j}^{2}\right) e^{i \beta_{j}}}{1-\rho_{j}^{2} e^{2 i \beta_{j}}} .
\end{aligned}
$$

Here, $\beta_{j}=k d_{j} v_{j}, v_{j}=\sqrt{\varepsilon_{j}}$ is a refractive index of the layers, and $k=2 \pi / \lambda$, where $\lambda$ denotes the free-space wavelength. The Fresnel interface coefficient $\rho_{j}$ is given by

$$
\rho_{j}=\frac{v_{j}-v_{b}}{v_{j}+v_{b}} .
$$

Given that our stack is composed of alternating layers, an odd $j$ subscript in $t_{j}$ and $r_{j}$ refers to layer type $A$, and an even value $j$ refers to layer type $B$. Equations (7)-(11) provide an exact description of the structure transmission and reflection for a given realization. They will be used for direct numerical calculations of the stack transmission logarithm and its mean using the Monte Carlo approach. The localization length does not depend on the background refractive index $v_{b}$, and it is assumed to be unity $\left(v_{b}=1\right)$ for the numerical calculations. In contrast the transmission length $\ell_{N}$ can depend on $v_{b}$ outside of the localized regime.

\section{B. Analytical treatment}

In the theoretical consideration we assume that the refractive index contrast between the layers is small $\left|\rho_{j}\right| \ll 1$; therefore the scattering is weak. The localization length of a random stack is an internal property of the stack and cannot depend on the refractive index of the medium where the stack is embedded (usually, free space $v_{b}=1$ ) [38]. Therefore in the analytical approach we assume $v_{b}=v_{B}$. To derive the expression for the localization length we adopt an approach similar to that in [22]. First, we linearize the recurrence relations

$$
\begin{gathered}
\ln T_{n}=\ln T_{n-1}+\ln t_{n}+R_{n-1} r_{n}, \\
R_{n}=r_{n}+R_{n-1} t_{n}^{2} .
\end{gathered}
$$

To obtain Eq. (12) we take the logarithm of the relation (7) and expand the logarithm of its denominator, while Eq. (13) is obtained by dropping the second term in the denominator of Eq. (8). In this approximation, we omit the terms which are proportional to the third-order products of the reflection coefficients $r_{j} r_{p} r_{q}$ and higher, given we consider weak scattering. Note that the expansion of the stack reflection $R_{n}$ in terms of the layer reflection coefficients $r_{j}$ contains only an odd number $r_{j}$ products, while the stack transmission $T_{n}$ contains only an even number of $r_{j}$ products. Therefore in this second-order approximation we include all terms of the $r_{j} r_{p}$ products and omit all higher-order even products.

Then, by summing the logarithmic transmission terms (12) in this approximation we obtain

$$
\ln T_{N}=\sum_{j=1}^{N / 2} \ln t_{2 j-1}+\sum_{j=2}^{N / 2} r_{2 j-1} \tilde{R}_{2 j-2},
$$

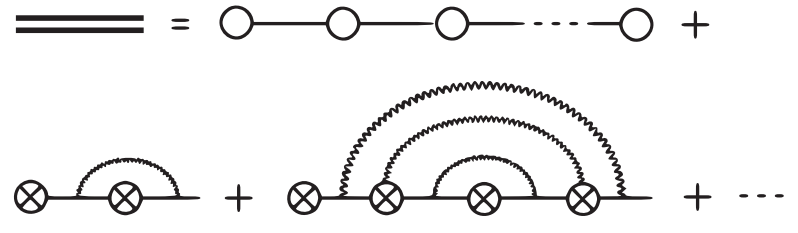

FIG. 2. Diagrammatic representation of the multiple scattering considered in (14) and (15). The first term in the diagram represents the logarithm of the direct transmission, while the second-order contribution is given by the sum of all "rainbow"-type diagrams (see the second line).

where $\tilde{R}_{2 j-2}$ is given by

$$
\tilde{R}_{2 j-2}=\sum_{m=1}^{2 j-3} r_{m} \prod_{p=m+1}^{2 j-2} t_{p}^{2} .
$$

Note that the direct expansion of the Dyson equation over the potential correlation functions is not applicable to the disorder distribution considered here. Also, even though the localization length is given by the stack transmittance, the localization length can be calculated by the logarithm of the stack transmission, which is a much simpler proposition.

The used second-order multiple-scattering approximation for the logarithm of the transmission can be represented by diagrams (see Fig. 2). The calculation of the localization length is given by the contribution of all "rainbow" diagrams and by the logarithm of the straight transmission. In Fig. 2 the slab reflection and transmission scattering are represented by crossed circles, propagation is represented by straight lines, and the correlated averages are indicated by the wiggly curves.

After substituting (15) into (14) and averaging, we obtain

$$
\left\langle\ln T_{N}\right\rangle=\frac{N}{2}\left\langle\ln t_{A}\right\rangle+\left\langle r_{A}\right\rangle^{2}\left\langle t_{B}^{2}\right\rangle \sum_{m=1}^{N / 2-1}\left(\frac{N}{2}-m\right) q^{m-1},
$$

where $q=\left\langle t_{A}^{2}\right\rangle\left\langle t_{B}^{2}\right\rangle$. The explicit forms of $\left\langle r_{A}\right\rangle,\left\langle t_{A}^{2}\right\rangle$, and $\left\langle\ln t_{A}\right\rangle$ are given by the relations (A1), (A5), and (A9), and the equation for $\left\langle t_{B}^{2}\right\rangle$ is obtained from (A5) by replacing $v_{A}$ with $v_{B}$.

The finite sum in (16) is an arithmetic-geometric progression which can be expressed in closed form. After the summation the expression for $\left\langle\ln T_{N}\right\rangle$ takes the form

$$
\begin{aligned}
\left\langle\ln T_{N}\right\rangle= & \frac{N}{2}\left\langle\ln t_{A}\right\rangle+\frac{\left\langle t_{B}^{2}\right\rangle\left\langle r_{A}\right\rangle^{2}}{2} \\
& \times\left[\frac{N}{1-q}+\frac{2\left(q^{N / 2}-1\right)}{(1-q)^{2}}\right] .
\end{aligned}
$$

After the substitution of (17) into (2) and taking the limit (3) the localization length in this weak-scattering limit takes the form

$$
-\frac{1}{\ell}=\operatorname{Re}\left[\frac{\left\langle\ln t_{A}\right\rangle}{2}+\frac{\left\langle r_{A}\right\rangle^{2}\left\langle t_{B}^{2}\right\rangle}{2(1-q)}\right],
$$

where the localization length $\ell$ is scaled by the mean of a layer thickness $\langle d\rangle$. The obtained equation for the localization length (18) is verified numerically in Sec. III. In the 
next section we use Eq. (18) to deduce the explicit analytic equations of the short- and long-wavelength asymptotes for the localization length $\ell$.

\section{Short- and long-wavelengths asymptotes of the localization length}

The obtained equation for the localization length (18) can be used to deduce the short- and long-wavelength asymptotes of the localization length. The explicit asymptotic forms of the localization length can be deduced from the short- and long-wavelength expansions of $\left\langle\ln t_{A}\right\rangle,\left\langle t_{A}^{2}\right\rangle,\left\langle t_{B}^{2}\right\rangle$, and $\left\langle r_{A}\right\rangle$. These expansions are derived in Appendixes A and B. In the subsequent presentation we scale the localization length and wavelength by the mean of a layer thickness $\langle d\rangle$ (5).

\section{Short-wavelength asymptotes}

To calculate the short-wavelength asymptote for the localization length $\ell$ it is necessary to calculate the shortwavelength asymptotes for the averages $\left\langle\ln t_{A}\right\rangle,\left\langle r_{A}\right\rangle,\left\langle t_{A}\right\rangle$, and $\left\langle t_{B}\right\rangle$. The details of these calculations are presented in Appendix A.

Substituting the obtained asymptotic expansions (A4), (A7), (A8), and (A11) into (18), the localization length at the short-wavelength limit takes the form

$$
\ell=\frac{2}{\rho_{A}^{2}} .
$$

Therefore the localization length $\ell$ scaled by the mean of the layer thickness $\langle d\rangle$ at short wavelengths tends to a constant which is defined by the square of the Fresnel interface coefficient $\rho_{A}$. This is typical behavior of the localization length at short wavelengths, where the localization length is determined by local scattering properties. In Sec. III the obtained shortwavelength asymptotic (19) is verified numerically.

\section{Long-wavelength asymptotes}

Here we present the explicit asymptotes for the localization length at the limit of long wavelengths. These asymptotes can be deduced from the long-wavelength asymptotes of averages $\left\langle\ln t_{A}\right\rangle,\left\langle r_{A}\right\rangle,\left\langle t_{A}\right\rangle$, and $\left\langle t_{B}\right\rangle$ derived in Appendix B. After the substitution of the long-wavelength asymptotes (B2)-(B4) into (18) the asymptotic expression for the localization length for $1<\alpha<2$ normalized to the mean thickness of a layer $\langle d\rangle$ takes the form

$$
\ell=-\frac{(\alpha / 2)^{\alpha-1}\left(v_{A}+v_{B}\right)^{2} \lambda^{\alpha}}{[2 \pi(\alpha-1)]^{\alpha} \Gamma(-\alpha) \cos (\pi \alpha / 2) \rho_{A}^{2}\left(v_{A}^{2} v_{B}^{\alpha}+v_{B}^{2} v_{A}^{\alpha}\right)},
$$

where $\Gamma(x)$ is the gamma function. Thus the localization length $\ell$ is proportional to $\lambda^{\alpha}$ at long wavelengths. Either this result has been established in the past numerically, or its general functional form has been derived. Here, we have presented the explicit expression for the localization length at long wavelengths. This algebraic dependence on wavelength $\ell \sim \lambda^{\alpha}$ is in contrast to the classical case where $\ell \sim \lambda^{2}$. We have verified the obtained long-wavelength asymptote (20) numerically in Sec. III.
To deduce the asymptotic equation for the localization length for $\alpha=2$ we substitute expansions (B7)-(B9) into (18). The localization length for $\alpha=2$ at long wavelengths takes the form

$$
\ell=\frac{\left(v_{A}+v_{B}\right)^{2} \lambda^{2}}{4 \pi^{2} v_{A}^{2} v_{B}^{2} \rho_{A}^{2}\left[\ln \lambda-\ln \left(\pi \sqrt{\nu_{A} v_{B}} e^{(\gamma+1 / 2)}\right)\right]},
$$

where $\gamma=0.517216$ is the Euler constant. At very long wavelengths expression (21) can be simplified

$$
\ell=\frac{\left(v_{A}+v_{B}\right)^{2}}{4 \pi^{2} v_{A}^{2} v_{B}^{2} \rho_{A}^{2}} \frac{\lambda^{2}}{\ln \lambda} .
$$

The functional form of (22) was reported previously [31] for systems with long-range correlation disorder. Note the obtained explicit asymptotic form of the localization length (22) is for the case in which the second moment does not exist. In Sec. III we verify the obtained asymptotic equation (21) numerically.

For the values $\alpha>2$ where the second moment for the distribution exists, the localization length takes the form

$$
\ell=\frac{\left(v_{A}+v_{B}\right)^{2} \lambda^{2}}{8 \pi^{2} v_{A}^{2} v_{B}^{2} \rho_{A}^{2} \operatorname{Var}(d)},
$$

where $\operatorname{Var}(d)$ denotes the variance of the distribution scaled by the squared mean of the layer thickness. Here we recover the classical result in which the localization length is proportional to $\lambda^{2}$ and inversely proportional to the variance of the disorder at long wavelengths. Equation (23) is deduced by substituting the long-wavelength asymptotes (B2)-(B4) into (18).

The obtained equations for the localization length can be used to determine the stack length of finite stacks to achieve localization.

\section{NUMERICAL RESULTS}

In this section we present numerical verification of the theoretical results presented in Sec. II. Presented below are the numerical Monte Carlo calculations in which we used $10^{4}$ realizations and we considered a stack composed of $N=$ $10^{5}$ layers. We also considered a higher/lower number of realizations and observed that fluctuations of the localization length are markedly less pronounced with a larger number of realizations. This is quite similar to cases where the second moment of disorder exists. We also observe that the localization length fluctuations are reduced with the increase of stack length, which is a demonstration of the self-averaging property of the localization length. This self-averaging requires longer stacks to take place for Levy disorder than for disorder distributions where the second moment exists. The refractive indices of layers are $v_{A}=1.4$ and $v_{B}=1.3$ in the presented calculations.

We use the exact recurrence relations (7) and (8) to calculate the stack transmission composed of $N$ layers for a single realization. In the numerical calculations we assumed that the stack is embedded in a uniform medium with the refractive index $v_{b}=1$. The widths of the layers are generated using the relation

$$
d_{j}=\frac{x_{m}}{u^{1 / \alpha}},
$$




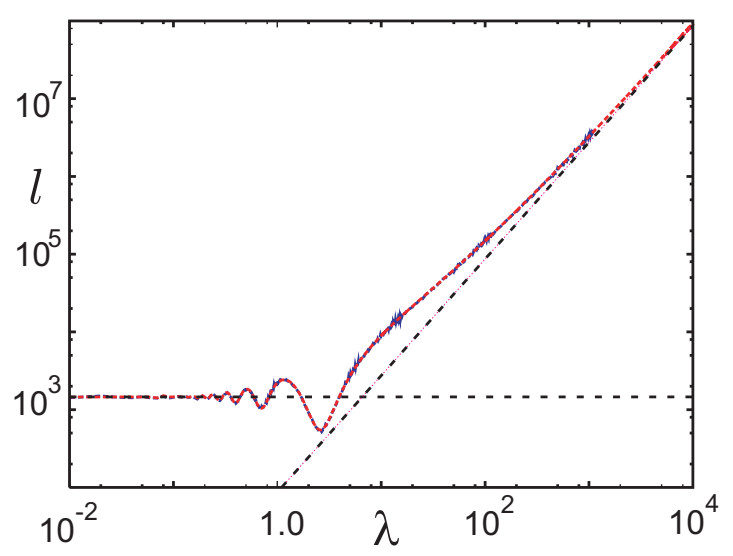

FIG. 3. Localization length $\ell$ as a function of wavelength $\lambda$ for a Levi stack with $\alpha=1.5$. The solid red curve is the analytical prediction (18), while the dotted blue curve is the numerical simulation. The horizontal dashed line is the derived short-wavelength asymptote (19), while the slanted dashed straight line is the long-wavelength asymptote (20).

where $u$ is a random variable uniformly distributed on the interval $[0,1]$. The obtained thickness values $d_{j}$ are distributed according to a Pareto distribution [39] (4). In all plots we scale the localization length and wavelength by the mean of the layer thickness $\langle d\rangle(5)$.

In Fig. 3 we present the localization length dependence on wavelength for $\alpha=1.5$ and $x_{m}=1 / 3$. Both the localization length $\ell$ and the wavelength $\lambda$ are scaled by the mean of the layer thickness $\langle d\rangle$. The blue dotted curve is the numerical calculation, while the red solid line is the theoretical description based on Eq. (18). There is excellent agreement between the curves. The curves are practically indistinguishable for all wavelengths.

At short wavelengths (see Fig. 3) the localization length tends to a constant. The horizontal dashed line is based on the short-wavelength asymptote (19). So at the short wavelength the localization length depends on the Fresnel reflection coefficient and on the mean layer thickness. The sloped dashed straight line in Fig. 3 is the long-wavelength asymptote based on Eq. (20). Therefore at long wavelengths the localization length is proportional to $\lambda^{\alpha}$, and the localization length has quite a complicated $\alpha$ dependence. The oscillatory behavior of the localization length for wavelengths $0.3<\lambda<2$ is associated with the band gap properties of the initial unperturbed periodic stack. In particular the minimums of the localization length are related to the band gaps, and maximum values are associated with the pass bands. In Fig. 4 we present the dependence of the localization length on wavelength for $\alpha=2$ and $x_{m}=0.5$. The solid red curve is the numerical calculation, while the blue dotted curve is the analytical description based on (18). The curves are indistinguishable except at very long wavelengths $\lambda>5000$. In order to characterize the localization length at such long wavelengths for this case it is required to consider longer stacks. We confirm this by numerical calculations.

The overall behavior of the localization length is similar to the case considered above. At short wavelengths the localization length tends to a constant given by relation (19)

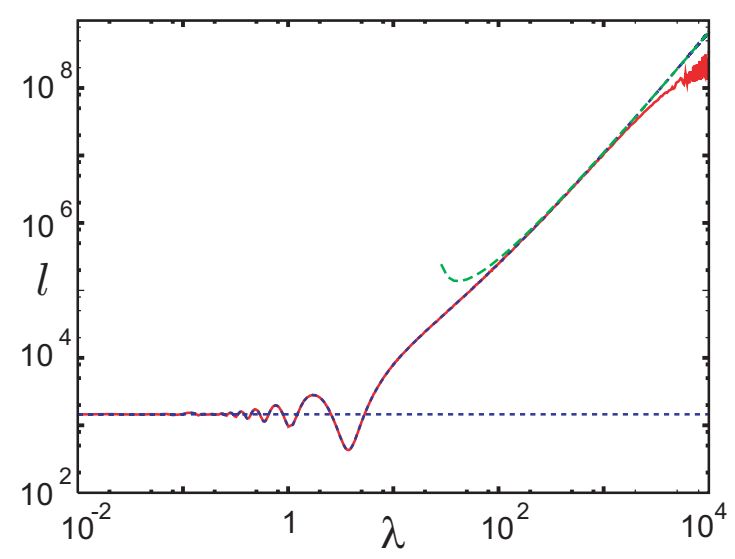

FIG. 4. Localization length $\ell$ as a function of $\lambda$ for a Levi stack for a critical value $\alpha=2$. The solid red curve is the numerical Monte Carlo calculation, while the dotted blue curve is the analytical prediction (18). The horizontal dashed line is the derived shortwavelength asymptote (19), while the green dashed curve is the long-wavelength asymptote (21).

(see the horizontal straight dotted line in Fig. 4), while the green dashed curve is the long-wavelength asymptote given by relation (21). This relation is applicable for $\lambda \gg$ $\pi \sqrt{v_{A} v_{B}} e^{(\gamma+1 / 2)}$. For the set of parameters used here this requirement leads to the condition $\lambda \gg 25$. The asymptotic expression given by (21) can be considered an intermediate asymptotic, while the long-wavelength asymptote is given by (22). Perhaps for practical applications the intermediate form of the long-wavelength asymptote given by (21) is more useful.

In Fig. 5 we plot the localization length as a function of wavelength for $\alpha=3.5$ and for $x_{m}=5 / 7$. For this set of parameters the second moment for the random distribution (4) exists, and we recover the classical behavior of the localization length $\sim \lambda^{2}$ at long wavelengths. The solid red curve (see Fig. 5) is the numerical calculation, and the dotted blue

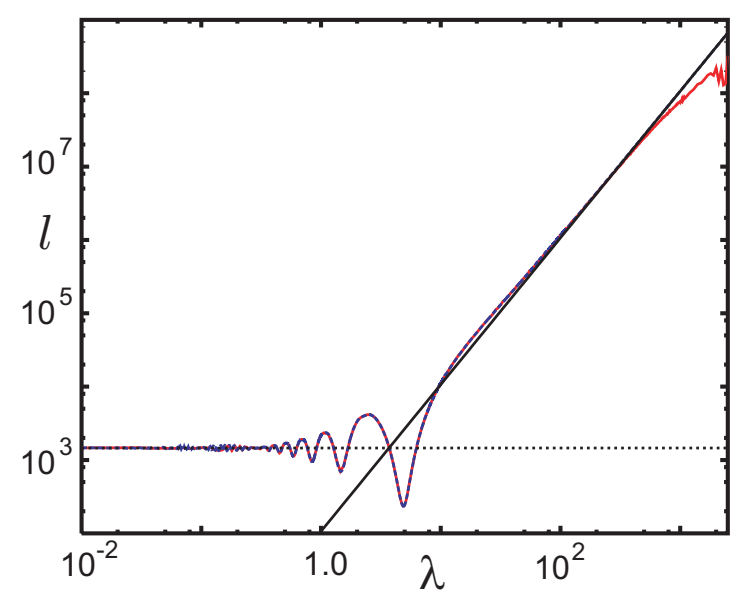

FIG. 5. Localization length $\ell$ as a function of wavelength $\lambda$ for a stack with $\alpha=3.5$. The solid red curve is the numerical simulation, while the dotted blue curve is the analytical prediction (18). The horizontal dotted line is the short-wavelength asymptote (19), while the slanted solid straight line is the long-wavelength asymptote (23). 
curve is the localization length calculation based on (18). We obtain excellent agreement between the curves except at long wavelengths, where the stack length is not sufficient to properly characterize the localization length. We confirm this by considering longer stacks.

The horizontal straight dotted line is the short-wavelength asymptotic description (19), while the slanted straight line is the long-wavelength asymptote given by (23). Therefore for $\alpha>2$ at long wavelengths the localization length is inversely proportional to the variance of disorder and directly proportional to $\lambda^{2}$.

\section{CONCLUSION}

Properties of the Anderson localization of classical waves in weakly scattering one-dimensional Levy glasses have been studied. In such media the variance of disorder does not exist, which strongly affects the localization properties in such systems. A general equation for the localization length (18) which is applicable for all wavelengths has been derived. The derived equation has been verified numerically, and it is in excellent agreement with the numerical Monte Carlo calculations. The explicit asymptotic expressions for the localization length for both short [Eq. (19)] and long [(20)-(23)] wavelengths have been deduced. These asymptotic expressions have been verified numerically. At short wavelengths the localization length tends to a constant defined by the Fresnel interface reflection coefficient and by the mean of the layer thickness. In this respect the localization length is not affected by the absence of the variance of the disorder distribution. This can be attributed to the fact that at such short wavelengths the localization length is determined by the local distribution of the parameters.

This is in stark contrast at long wavelengths, where for $1<$ $\alpha<2$ the localization length has an algebraic dependence, $\ell \sim \lambda^{\alpha}$, and for $\alpha=2$ it has a transcendental behavior, $\ell \sim$ $\lambda^{2} / \ln \lambda$. Such a functional form of the localization length was predicted first in [31] for a different system with a long correlation disorder. It would be interesting to know if this transcendental behavior takes place for other systems as well.

Although here we have confined ourselves to the case of only layer thickness disorder, the derived equation (18) can be applied to systems with refractive index disorder or systems with both refractive index and thickness disorders simultaneously. The obtained equation is applicable for offaxis incidence as well. The presented results could be useful for electronic systems with a power law decaying impurity potential also.

\section{ACKNOWLEDGMENT}

This research was supported by the Australian government through the Australian Research Council (Project No. DP150102758).

\section{APPENDIX A: DETAILS OF AVERAGING AT THE SHORT-WAVELENGTH LIMIT}

In this appendix we present the short-wavelength asymptotic expansion calculations for averages $\left\langle\ln t_{A}\right\rangle,\left\langle r_{A}\right\rangle,\left\langle t_{A}\right\rangle$, and $\left\langle t_{B}\right\rangle$. At the short-wavelength limit $k \rightarrow \infty$ these averages can be calculated by the repeated application of integration by parts. The average of $\left\langle r_{A}\right\rangle$ can be written as

$$
\left\langle r_{A}\right\rangle=\int_{x_{m}}^{\infty} \frac{\rho_{A}\left(1-e^{i 2 k v_{a} x}\right)}{1-\rho_{A}^{2} e^{i 2 k v_{a} x}} \frac{\alpha x_{m}^{\alpha}}{x^{\alpha+1}} d x .
$$

After changing the integration variable $x=x_{m} t$, expanding the denominator into a geometric series, and changing the order of summation and integration, we obtain

$$
\left\langle r_{A}\right\rangle=\rho_{A}+\alpha \sum_{n=1}^{\infty}\left(\rho_{A}^{2 n+1}-\rho_{A}^{2 n-1}\right) \int_{1}^{\infty} \frac{e^{i a n t}}{t^{\alpha+1}} d t,
$$

where $a=2 k v_{A} x_{m}$. The repeated application of integration by parts to the integral in (A2) leads to the expansion

$$
\begin{aligned}
\int_{1}^{\infty} \frac{e^{i a n t}}{t^{\alpha+1}} d t= & -\frac{e^{i a n}}{i n a}-(\alpha+1) \frac{e^{i a n}}{(\text { ina })^{2}} \\
& +\frac{(\alpha+1)(\alpha+2)}{(\text { ian })^{2}} \int_{1}^{\infty} \frac{e^{i a n t}}{t^{\alpha+3}} d t .
\end{aligned}
$$

The first term in this expansion is the dominant term at the short-wavelength limit $a \rightarrow \infty$. Therefore we deduce

$$
r_{A} \approx \rho_{A} \text {. }
$$

The mean for $\left\langle t_{A}^{2}\right\rangle$ is given by

$$
\left\langle t_{A}^{2}\right\rangle=\int_{x_{m}}^{\infty} \frac{\left(1-\rho_{A}^{2}\right) e^{i k v_{a} x}}{1-\rho_{A}^{2} e^{i 2 k v_{a} x}} \frac{\alpha x_{m}^{\alpha}}{x^{\alpha+1}} d x .
$$

After the expansion of the fraction in (A5) into a geometric series we obtain

$$
\left\langle t_{A}^{2}\right\rangle=\alpha\left(1-\rho_{A}^{2}\right)^{2} \sum_{n=1}^{\infty} n \rho_{A}^{2 n-2} \int_{1}^{\infty} \frac{e^{i 2 k n v_{A} x_{m} t}}{t^{\alpha+1}} d t .
$$

Taking into account the asymptotic expansion (A3), the dominant term of $\left\langle t_{A}^{2}\right\rangle$ at short wavelengths is

$$
\left\langle t_{A}^{2}\right\rangle \approx-\frac{\alpha\left(1-\rho_{A}^{2}\right)^{2}}{i a} \frac{e^{i a}}{1-\rho_{A}^{2} e^{i a}} .
$$

The short-wavelength asymptote for $\left\langle t_{B}^{2}\right\rangle$ can be deduced from (A7) by replacing $\rho_{A}$ with zero and $v_{A}$ with $v_{B}$,

$$
\left\langle t_{B}^{2}\right\rangle \approx-\frac{\alpha e^{i b}}{i b},
$$

where $b=2 k x_{m} v_{B}$.

The short-wavelength expansion for $\left\langle\ln t_{A}\right\rangle$ can be obtained in a similar way. The average of $\left\langle\ln t_{A}\right\rangle$ takes the form

$$
\left\langle\ln t_{A}\right\rangle=\ln \left(1-\rho_{A}^{2}\right)+i k v_{A} d-\alpha \int_{1}^{\infty} \ln \left(1-\rho_{A}^{2} e^{i a t}\right) \frac{d t}{t^{\alpha+1}} .
$$

After the expansion of the integral in (A9) into a power series we obtain

$$
\left\langle\ln t_{A}\right\rangle=\ln \left(1-\rho_{A}^{2}\right)+i k v_{A} d+\alpha \sum_{n=1}^{\infty} \frac{\rho_{A}^{2 n}}{n} \int_{1}^{\infty} \frac{e^{i a n t} d t}{t^{\alpha+1}} .
$$


Therefore the real part of $\left\langle\ln t_{A}\right\rangle$ at short wavelengths can be approximated by

$$
\operatorname{Re}\left\langle\ln t_{A}\right\rangle \approx \ln \left(1-\rho_{A}^{2}\right) \approx=-\rho_{A}^{2} .
$$

After substitution of the obtained asymptotes (A4), (A7), (A8), and (A11) into (18) we deduce the short-wavelength asymptote for the localization length (19).

\section{APPENDIX B: DETAILS OF AVERAGING AT THE LONG-WAVELENGTH LIMIT $\boldsymbol{k} \rightarrow \boldsymbol{0}$}

The long-wavelength asymptotes for the mean of $\left\langle\ln t_{A}\right\rangle$, $\left\langle r_{A}\right\rangle$, and $\left\langle t_{A, B}^{2}\right\rangle$ can be found using the following series expansion [40] for integral (A3):

$$
\int_{1}^{\infty} \frac{e^{i a n t}}{t^{\alpha+1}} d t=\alpha a^{\alpha} n^{\alpha} \Gamma(-\alpha) e^{-i \pi \alpha / 2}+\sum_{m=0}^{\infty} \frac{(i a n)^{m}}{m !(-m+\alpha)},
$$

where $\Gamma(x)$ is the gamma function. This expansion is applicable for only fractional $\alpha$. After the substitution of (B1) into (A2), (A6), and (A10) the long-wavelength asymptotes for $\left\langle\ln t_{A}\right\rangle,\left\langle r_{A}\right\rangle$, and $\left\langle t_{A, B}^{2}\right\rangle$ take the forms

$$
\begin{aligned}
& \left\langle\ln t_{A}\right\rangle \approx \alpha a^{\alpha} \Gamma(-\alpha) e^{-i \alpha \pi / 2} \rho_{A}^{2}+\frac{i \alpha a \rho_{A}^{2}}{\alpha-1}+\frac{\alpha a^{2} \rho_{A}^{2}}{2(2-\alpha)}, \\
& \left\langle r_{A}\right\rangle \approx-\alpha a^{\alpha} \Gamma(-\alpha) \rho_{A} e^{-i \alpha \pi / 2}+\frac{i \alpha a \rho_{A}}{\alpha-1}-\frac{\alpha a^{2} \rho_{A}}{2(2-\alpha)}, \\
& \left\langle t_{A}^{2}\right\rangle \approx 1+\alpha a^{\alpha} \Gamma(-\alpha) e^{-i \alpha \pi / 2}+\frac{i \alpha a}{\alpha-1}+\frac{\alpha a^{2} \rho_{A}}{2(2-\alpha)} .
\end{aligned}
$$

The value $\left\langle t_{B}^{2}\right\rangle$ can be deduced by substituting $\rho_{A}=0$ and replacing $\left\langle t_{A}^{2}\right\rangle$ with $\left\langle t_{B}^{2}\right\rangle$ and $v_{A}$ with $v_{B}$ in (B4).
For $\alpha=2$ the integral (B1) can be calculated in closed form,

$$
\begin{aligned}
\int_{1}^{\infty} \frac{e^{i a n t}}{t^{3}} d t= & \frac{1}{2}(1+i a n) e^{i a n}-\frac{i \pi a^{2} n^{2}}{4} \\
& +\frac{a^{2} n^{2}}{2}[\operatorname{Ci}(a n)+i \operatorname{Si}(a n)],
\end{aligned}
$$

where $\operatorname{Ci}(x)$ and $\operatorname{Si}(x)$ are integral sine and cosine functions given by

$$
\begin{aligned}
\operatorname{Si}(x) & =\int_{0}^{x} \frac{\sin t}{t} d t, \\
\operatorname{Ci}(x) & =-\int_{x}^{\infty} \frac{\cos t}{t} d t .
\end{aligned}
$$

The short-wavelength asymptote for (B5) can be readily deduced:

$$
\int_{1}^{\infty} \frac{e^{i a n t}}{t^{3}} d t \approx \frac{1}{2}+i a n+\frac{a^{2} n^{2}}{2}\left(\ln (a n)+\gamma-\frac{3}{2}-\frac{i \pi}{2}\right),
$$

where $\gamma \approx 0.517216$ is the Euler constant. The longwavelength asymptotes for $\left\langle\ln t_{A}\right\rangle,\left\langle r_{A}\right\rangle$, and $\left\langle t_{A, B}^{2}\right\rangle$ are then expressed as

$$
\begin{gathered}
\left\langle\ln t_{A}\right\rangle \approx \rho_{A}^{2} a^{2}\left(\ln a+\gamma-\frac{3}{2}\right), \\
\left\langle r_{A}\right\rangle \approx-\rho_{A}\left(a^{2}\left(\ln a \gamma-\frac{3}{2}\right)+2 i a-\frac{i \pi a^{2}}{2}\right), \\
\left\langle t_{A}^{2}\right\rangle \approx 1+a^{2}\left(\ln a \gamma-\frac{3}{2}\right)+2 i a-\frac{i \pi a^{2}}{2} .
\end{gathered}
$$

The value of $\left\langle t_{B}^{2}\right\rangle$ can be deduced in a way similar to that for $1<\alpha<2$. By using the obtained asymptotic expansions (B2)-(B4) the long-wavelength asymptotes for the localization length (20) are obtained from (18) for $1<\alpha<2$. Using the expansions (B7)-(B9), the long-wavelength localization length asymptote (21) for $\alpha=2$ is deduced from (18).
[1] P. W. Anderson, Phys. Rev. 109, 1492 (1958).

[2] S. John, Phys. Rev. Lett. 53, 2169 (1984).

[3] I. M. Lifshits, S. A. Gredeskul, and L. A. Pastur, Introduction to the Theory of Disordered Systems (Wiley, New York, 1987), and references therein.

[4] P. Sheng, Scattering and Localization of Classical Waves in Random Media (World Scientific, Singapore, 1991).

[5] V. D. Freilikher and S. A. Gredeskul, Prog. Opt. 30, 137 (1992).

[6] P. Sheng, Introduction to Wave Scattering, Localization, and Mesoscopic Phenomena (Springer, Heidelberg, 2006).

[7] P. Marcos and C. M. Soukoulis, Wave Propagation from Electrons to Photonic Crystals and Left-Handed Materials (Princeton University Press, Princeton, NJ, 2008).

[8] N. F. Mott and W. D. Twose, Adv. Phys. 10, 107 (1961).

[9] H. Furstenberg, Trans. Am. Math. Soc. 108, 377 (1963).

[10] 50 Years of Anderson Localization, edited by E. Abrahams (World Scientific, London, 2010).

[11] S. John, Phys. Rev. B 31, 304 (1985).
[12] V. D. Freilikher, M. Pustilnik, and I. Yurkevich, Phys. Rev. Lett. 73, 810 (1994).

[13] J. C. J. Paasschens, T. S. Misirpashaev, and C. W. J. Beenakker, Phys. Rev. B 54, 11887 (1996).

[14] A. A. Asatryan, N. A. Nicorovici, L. C. Botten, C. M. de Sterke, P. A. Robinson, and R. C. McPhedran, Phys. Rev. B 57, 13535 (1998).

[15] B. Derrida and E. Gardner, J. Phys. (Paris) 45, 1283 (1984).

[16] B. A. van Tiggelen and A. Tip, J. Phys. I 1, 1145 (1991).

[17] A. A. Asatryan, L. C. Botten, M. A. Byrne, V. D. Freilikher, S. A. Gredeskul, I. V. Shadrivov, R. C. McPhedran, and Y. S. Kivshar, Phys. Rev. Lett. 99, 193902 (2007).

[18] P. Han, C. T. Chan, and Z. Q. Zhang, Phys. Rev. B 77, 115332 (2008).

[19] A. J. Chaves, N. M. R. Peres, and F. A. Pinheiro, Phys. Rev. B 92, 195425 (2015).

[20] M. Inoue and T. Fujii, J. Appl. Phys. 81, 5659 (1997).

[21] V. Baluni and J. Willemsen, Phys. Rev. A 31, 3358 (1985). 
[22] A. A. Asatryan, S. A. Gredeskul, L. C. Botten, M. A. Byrne, V. D. Freilikher, I. V. Shadrivov, R. C. McPhedran, and Y. S. Kivshar, Phys. Rev. B 81, 075124 (2010).

[23] E. J. Torres-Herrera, F. M. Israilev, and N. M. Makarov, Eourophys. Lett. 98, 27003 (2012).

[24] E. Reyes-Gomez, A. Bruno-Alfonso, S. B. Cavalcanti, and L. E. Oliveira, Phys. Rev. B 85, 195110 (2012).

[25] D. Applebaum, Levy Processes and Stochastic Calculus (Cambridge University Press, Cambridge, 2004).

[26] D. J. Thoules, J. Phys. C 5, 77 (1972).

[27] P. Lloyd, J. Phys. C 2, 1717 (1969).

[28] F. Falceto and V. A. Gopar, Europhys. Lett. 92, 57014 (2010).

[29] P. R. Wells, Jr., J. d'Albuquerquee e Castro, and S. L. A. de Queiroz, Phys. Rev. B 78, 035102 (2008).

[30] A. A. Fernandez-Marin, J. A. Mendez-Bermudez, and V. A. Gopar, Phys. Rev. A 85, 035803 (2012).

[31] S. John and M. J. Stephen, Phys. Rev. B 28, 6358 (1983).
[32] D. Mogilevtsev, F. A. Pinheiro, R. R. dos Santos, S. B. Cavalcanti, and L. E. Oliveira, Phys. Rev. B 84, 094204 (2011).

[33] F. M. Izrailev and N. M. Makarov, Phys. Rev. Lett. 102, 203901 (2009).

[34] F. M. Izrailev, A. A. Krokhin, and N. M. Makarov, Phys. Rep. 512, 125 (2012).

[35] A. G. Ardakani, Eur. Phys. J. B 89, 75 (2016).

[36] P. Barthelemy, J. Bertolloti, and D. S. Wiersma, Nature (London) 453, 495 (2008).

[37] S. S. Zakeri, S. Lepri, and D. S. Wiersma, Phys. Rev. E 91, 032112 (2015).

[38] A. A. Asatryan, L. C. Botten, M. A. Byrne, V. D. Freilikher, S. A. Gredeskul, I. V. Shadrivov, R. C. McPhedran, and Y. S. Kivshar, Phys. Rev. B 85, 045122 (2012).

[39] H. Tanizaki, Computational Methods in Statistics and Econometrics (Marcel Dekker, New York, 2004).

[40] Wolfram Research, Inc., Mathematica, version 11.01 (Wolfram Research, Inc., Champaign, IL, 2014). 